\title{
Using 'Bill and Keep' Interconnect Arrangements to Soften Network Competition
}

\author{
by \\ Joshua S. Gans and Stephen P. King* \\ University of Melbourne \\ $10^{\text {th }}$ March, 2000
}

This paper demonstrates that low (below marginal cost) interconnect or access charges can be used to sustain high subscription prices in an environment of network competition with two-part tariffs and price discrimination. This result stands in contrast to other results in the literature suggesting that high interconnect charges can play a collusive role. Journal of Economic Literature Classification Numbers: L41, L96.

Keywords. network competition, interconnection, bill and keep, and two part tariffs.

\footnotetext{
* Melbourne Business School and Department of Economics, respectively. All correspondence to Joshua Gans, Email: J.Gans@mbs.unimelb.edu.au. We would like to thank Patrick Rey and Julian Wright for their comments on an earlier version of this paper. The latest version of this paper is available at: www.mbs.unimelb.edu.au/jgans.
} 
US and European reform in local, fixed-line telecommunications has highlighted the potential for network competition. A key element in competition is the terms that govern interconnection between competing networks. These pricing arrangements between networks will influence the costs of making inter- and sometimes intra-network calls and hence, the level of effective competition.

A number of recent papers have raised concerns that interconnect fees between competing networks can be used to facilitate collusive outcomes. Laffont, Rey and Tirole (1998a), Armstrong (1998) and Carter and Wright (1999) each demonstrate that, by negotiating high interconnect charges, monopoly outcomes can result despite the existence of competing networks. Interconnect charges play an important role in the (marginal) cost of calls. Under a caller-pays system, they represent the amount a network that originates a call pays to the network that terminates a call. Consequently, the higher the interconnect charge, the higher are marginal call costs and hence, the price of calls. By agreeing to a high enough interconnect charge, networks can achieve a monopoly call price and, because flows of inter-network traffic tend to be symmetric (Williams, 1995), neither bears any burden from the high charges to each other.

Two important assumptions underlie this outcome. First, price discrimination between intra- and inter-network calls is not possible. This might reflect regulatory restrictions or, given the existence of local number portability, reflect that consumers are not aware of the network they are calling. This means that interconnect fees enter directly into the costs of all calls and not simply inter-network calls. ${ }^{1}$ Second, consumers face simple linear prices set on a per call or per call minute basis. If this

\footnotetext{
${ }^{1}$ Laffont, Rey and Tirole (1998b) show that this assumption is not necessary for high interconnection charges to be used to raise call prices.
} 
second assumption were relaxed (say to allow for two-part tariffs), then high interconnect fees would lead to high call prices but at a loss of consumer value. This, in turn would tend to lower network profits. In this environment, under any reciprocal interconnect arrangement (where each network pays each other the same interconnect fee), networks would either be indifferent regarding interconnect fee levels (Laffont, Rey and Tirole, 1998a) or prefer a lower fee (Laffont, Rey and Tirole, 1998b).

In cellular or mobile communications and many Internet and data services, networks distinguish between inter- and intra-network calls, providing information or price differentials to consumers. Moreover, the pricing structures are increasingly non-linear. This is in contrast to local phone networks that map more closely into the environment discussed by previous researchers. In this paper, we demonstrate that under price discrimination and non-linear pricing, competing networks will wish to negotiate very low interconnect charges. These will often be below marginal cost and may involve a 'bill and keep' or zero charge, or perhaps even a negative charge. These low charges will be used to soften price competition between networks.

Laffont, Rey and Tirole (1998b) analyse this case. They conclude that interconnect charges will be negotiated to equal the marginal cost of terminating a call (or connection). We demonstrate here that their conclusion is incorrect and, invariably, the negotiated interconnect charge will lie below marginal cost. The effect of this is to make networks' termination revenues (i.e., the amount they receive from incoming calls) less than termination costs. This means that those networks will face fewer benefits from lowering charges to attract marginal subscribers and will soften their bidding for all consumers (i.e., will offer higher fixed fees). This raises both networks' profits. Hence, the Laffont, Rey and Tirole (1998b) model actually supports the use of low, rather than high, interconnect fees to achieve more collusive outcomes. 


\section{Model Set-Up}

We utilize the same set-up as Laffont, Rey and Tirole (1998b). Suppose there are two networks, $A$ and $B$. Each faces the same costs. The cost of connecting a customer is $f$ while the marginal cost of terminating or originating a call is $c_{0}$ and the marginal trunk cost of a call is $c_{1}$. Thus, the total marginal cost of a call is $c \equiv 2 c_{0}+c_{1}$.

The two networks compete for a customer set of measure 1. Each network is assumed to have full coverage. We suppose that the two networks sell a differentiated but substitutable product. This is modelled by assuming that each network is located at either end of a line of length 1 with $A$ located at 0 and $B$ located at 1 . Consumers are located uniformly over the line. Given income $y$ and calls made $q$, a consumer located at $x$ and joining network $i$ has utility:

$$
y+v_{0}-t\left|x-x_{i}\right|+u(q)
$$

where $v_{0}$ represents a consumer's intrinsic value of subscribing to some network and $t\left|x-x_{i}\right|$ denotes the cost of subscribing to a network with 'address' $x_{i}(i=A, B){ }^{2}$

To consider the determinants of consumer choice between carriers, let $v(p)$ be the indirect utility function of a consumer who faces an outgoing call price of $p$ (per call or call-minute). In other words, $v(p) \equiv \max _{q} u(q)-p q$. We interpret this, as do Laffont, Rey and Tirole (1998b), as the utility generated from calling a particular person. Implicitly, this means that consumers do not refrain from calling people when the price of doing so rises but merely that they call them less often or make shorter calls.

\footnotetext{
2 It is assumed that $y$ and $v_{0}$ are sufficiently high that a consumer always wants to subscribe to one network.
} 
Consumers can be offered non-linear prices, i.e., a two-part tariff; $W_{i}\left(q_{i}, q_{i j}\right)=F_{i}+p_{i} q_{i}+p_{i j} q_{i j}$.

where:

- $F_{i}$ is the fixed fee or subscriber charge;

- $\quad p_{i}$ is the per call charge from network $i$;

- $q_{i}$ is the quantity of calls to network $i$;

- $\quad p_{i j}$ is the per call charge from network $i$ to network $j$

- $\quad q_{i j}$ is the quantity of calls from network $i$ to network $j$.

The value to a consumer subscribing to network $A$ or $B$ is, respectively,

$$
\begin{aligned}
& V_{A}=\alpha v\left(p_{A}\right)+(1-\alpha) v\left(p_{A B}\right)-F_{A} \\
& V_{B}=\alpha v\left(p_{B A}\right)+(1-\alpha) v\left(p_{B}\right)-F_{B}
\end{aligned}
$$

where $\alpha$ is the market share of network $A$. Notice that this specification implicitly includes our assumption that consumers choose their calling patterns among networks randomly; save for differing call prices.

The market share of network $A, \alpha$, is determined by the point of indifference between $A$ and $B$. That is,

$$
\begin{aligned}
& V_{A}-t \alpha=V_{B}-t(1-\alpha) \\
& \Rightarrow \alpha=\frac{1}{2}+\sigma\left(V_{A}-V_{B}\right)
\end{aligned}
$$

where $\sigma=1 /(2 t)$ is the degree of substitutability between the two mobile networks.

When bidding for customers, each network will find it optimal to set each call charge equal to its marginal cost. Let $T_{i j}$ be the termination charge from network $i$ to network $j$. Focusing on network $A$, the usage prices are: $p_{A}=c$ and $p_{A B}=c_{1}+c_{0}+T_{B A}$. This is a common outcome when firms can set multi-part tariffs. When price is set equal to marginal cost, the sum of consumer surplus and that 
network's per customer profits is maximised. If this were the only component of pricing, the network would not recover any fixed costs such as customer connection costs. However, the fixed charge, $F_{A}$, can be utilised to divide total value created (that is, utility less costs).

Given that usage charges are set equal to a network's marginal cost for various services, the market share of network $A$ becomes:

$$
\alpha=\frac{\frac{1}{2}+\sigma\left(v\left(c_{1}+c_{0}+T_{B A}\right)-v(c)+F_{B}-F_{A}\right)}{1-\sigma\left(2 v(c)-v\left(c_{1}+c_{0}+T_{B A}\right)-v\left(c_{1}+c_{0}+T_{A B}\right)\right)}
$$

Note that an interior equilibrium exists if $\frac{1}{\sigma}>2 v(c)-v\left(c_{1}+c_{0}+T_{B A}\right)-v\left(c_{1}+c_{0}+T_{A B}\right)$; that is, so long as termination charges and the degree of substitution are not too high.

\section{Equilibrium Subscription Fees}

We now consider each network's (simultaneous) choice of subscription fees; $F_{A}$ and $F_{B}$ for $A$ and $B$, respectively; and look for a Nash equilibrium. Given the usage charges network A's profit is,

$$
\pi_{A}=\alpha\left(F_{A}-f\right)+\alpha(1-\alpha)\left(T_{A B}-c_{0}\right) q_{B A} .
$$

This profit is the sum of the network's profits from its own customers and the profit from terminating calls from the other network. $A$ will choose $F_{A}$ to maximise these profits, yielding the first order condition:

$$
\frac{\partial \pi_{A}}{\partial F_{A}}=\alpha+\frac{\partial \alpha}{\partial F_{A}}\left(F_{A}-f+(1-2 \alpha)\left(T_{A B}-c_{0}\right) q_{B A}\right)=0
$$

where

$$
\frac{\partial \alpha}{\partial F_{A}}=-\frac{\sigma}{1-\sigma\left(2 v(c)-v\left(c_{1}+c_{0}+T_{B A}\right)-v\left(c_{1}+c_{0}+T_{A B}\right)\right)}
$$


Raising its fixed charge increases the direct revenues $A$ earns from its inframarginal customers. However, it also drives away marginal customers. This is costly because not only does $A$ loose direct revenues from these customers, but also the termination revenues it would otherwise receive when subscribers on other networks call those customers. $B$ faces the same trade-off in determining its pricing.

The existence of termination revenues makes a network with less than half of the customers a tougher price competitor. When networks discount to gain a customer, they attract the (net) calls from other networks to that customer and receive the resultant termination revenues. These revenues depend on the market share of each network, and are maximised when networks are of equal size, so that a network with a market share below one half always finds the marginal customer more valuable and intensely bids for that customer.

So long as $\sigma$ is low enough (i.e., networks are relatively poor substitutes) and $T_{A B}$ and $T_{B A}$ are not too far above $c_{0}$ (i.e., interconnection profits are not too large), then there exists a unique (Nash) equilibrium. ${ }^{3}$ The equilibrium market share of network $A$ is:

$\alpha=\frac{1}{2}+\sigma \frac{\frac{1}{2}\left(v\left(c_{1}+c_{0}+T_{B A}\right)-v\left(c_{1}+c_{0}+T_{A B}\right)\right)}{3\left(1-\sigma\left(2 v(c)-v\left(c_{1}+c_{0}+T_{B A}\right)-v\left(c_{1}+c_{0}+T_{A B}\right)-2\left(T_{A B}+T_{B A}-2 c_{0}\right) q_{B A}\right)\right.}$

Notice that when $T_{A B}=T_{B A}, \alpha$ is equal to $1 / 2$. When $T_{A B}>T_{B A}, \alpha \geq \frac{1}{2}$. Higher termination fees by network $A$ are reflected in $B$ 's price to its customers for offnetwork calls. By raising its interconnection fees, network $A$ places its competitor at a disadvantage and raises its market share.

${ }^{3}$ With equilibrium subscription fee for network $i$ :

$F_{i}=f-\frac{2 \sigma q_{j i} T_{i j}\left(2 \sigma\left(v(c)-v\left(c_{0}+c_{1}+T_{i j}\right)-1\right)+\left(\sigma\left(2 v(c)-v\left(c_{0}+c_{1}+T_{j i}\right)-v\left(c_{0}+c_{1}+T_{i j}\right)-1\right)\left(3+2 \sigma q_{i j} T_{i j}-6 \sigma v(c)+2 \sigma v\left(c_{0}+c_{1}+T_{i j}\right)+4 \sigma v\left(c_{0}+c_{1}+T_{j i}\right)\right)+2 \sigma c_{0}\left(q_{i j}\left(1-2 \sigma v(c)+\sigma v\left(c_{0}+c_{1}+T_{i j}\right)+\sigma v\left(c_{0}+c_{1}+T_{j i}\right)\right)+q_{j i}\left(1-2 \sigma v(c)+2 \sigma v\left(c_{0}+c_{1}+T_{j i}\right)\right)\right.\right.\right.}{2 \sigma\left(3-2 \sigma\left(c_{0}\left(q_{i j}+q_{j i}\right)-q_{j i} T_{i j}-q_{i j} T_{j i}\right)-3 \sigma\left(2 v(c)-v\left(c_{0}+c_{1}+T_{i j}\right)-v\left(c_{0}+c_{1}+T_{j i}\right)\right)\right)}$ 


\section{Interconnection Charges}

We begin by considering what happens when $A$ and $B$ set their termination charges independently. While a precise solution is not possible we can demonstrate the following:

Proposition 1. In any symmetric Nash equilibrium, $T_{A B}=T_{B A}>c_{0}$.

PROOF: $A$ 's profits (taking into account the interior equilibrium values of $F_{A}$ and $F_{B}$ ) are:

$$
\pi_{A}=\frac{1+\sigma\left(T_{A B}-c_{0}\right) q_{B A}-\sigma \Lambda}{4 \sigma}\left(1+2 \sigma \frac{\Omega+\frac{1}{2} \Lambda}{2 \sigma\left(T_{A B}+T_{B A}-2 c_{0}\right) q_{B A}+3(1-\sigma \Lambda)}\right)^{2}
$$

where

$$
\Lambda=2 v(c)-v\left(c_{1}+c_{0}+T_{B A}\right)-v\left(c_{1}+c_{0}+T_{A B}\right)
$$

$\Omega=v\left(c_{1}+c_{0}+T_{B A}\right)-v(c)$. Taking the derivative of this with respect to $T_{A B}$ and setting $T_{A B}=T_{B A}=c_{0}$, we have: $-\frac{1}{6} v^{\prime}(c)>0$ implying that at this point $A$ (and symmetrically $B$ ) would increase its profits by raising $T_{A B}\left(T_{B A}\right)$.

This outcome is akin to the standard double marginalisation result that arises when firms set prices for complementary services independently. What this means is that prices are higher than they otherwise would be and profits and consumer surplus are lower.

Consumer surplus is reduced because the price for calls between networks is too high and hence, fewer calls are made. This also raises the possibility that a symmetric equilibrium may not exist. Termination charges set above cost mean that the network with a greater number of subscribers will face lower average call costs. If networks were close substitutes this may lead to a corner solution with all subscribers on a single network. Here we have ruled out this possibility by our assumption that networks are not close substitutes so that the equilibrium remains interior.

We now consider the interconnect fees that arise when these are chosen cooperatively through negotiations rather than non-cooperatively. Following the notation of Laffont, Rey and Tirole (1998b), let $T_{A B}=T_{B A}=m c+c_{0}$ and suppose that 
networks will negotiate a choice of $m$ to maximise their joint profits. $m$ is the mark-up on interconnect charges relative to total call costs. In a symmetric equilibrium, network profit becomes:

$$
\pi_{i}=\frac{1}{4 \sigma}-\frac{1}{4}(2(v(c)-v((1+m) c))-m c q((1+m) c))
$$

This is the profit equation derived by Laffont, Rey and Tirole (1998b, p.53) where $q_{A B}=q_{B A}=q((1+m) c)$. Note that when $m=0$, the equilibrium profits of each network are their Hotelling level, $\pi^{H}=\frac{1}{4 \sigma}$, that would arise in competition where customers had unit demands. ${ }^{4}$

Given this, we can prove the following proposition:

Proposition 2. The negotiated interconnect arrangement has $m<0$ with negotiated profits exceeding $\pi^{H}$.

PROOF: Taking the derivative of (5) with respect to $m$, we have:

$$
\frac{\partial \pi_{i}}{\partial m}=\frac{1}{2} c v^{\prime}((1+m) c)+\frac{1}{4} c q((1+m) c)+\frac{1}{2} m c^{2} q^{\prime}((1+m) c)
$$

Noting that, by Shepherd's Lemma, $v^{\prime}=-q$ gives:

$$
\frac{\partial \pi_{i}}{\partial m}=-\frac{1}{4} c q((1+m) c)+\frac{1}{4} m c^{2} q^{\prime}((1+m) c)
$$

This is strictly less than zero when $m \geq 0$ so that the optimal level of $m$ is strictly less than zero. The optimal value of $m$ is given implicitly by $m^{*}$, where $q\left(\left(1+m^{*}\right) c\right)=m c q^{\prime}\left(\left(1+m^{*}\right) c\right)$.

To check that $m^{*}$ represents a level of maximum profits note that $\frac{\partial^{2} \pi}{\partial m^{2}}=\frac{1}{4} m c^{3} q^{\prime \prime}((1+m) c)$. This will be negative if $m c^{3} q^{\prime \prime}((1+m) c)<0$. Note that:

(1) $\partial^{2} \pi / \partial m^{2}=0$ when $m=0$. 
(2) if $q^{\prime \prime}>0$ then $\partial^{2} \pi / \partial m^{2}>0$ when $m>0$, and $\partial^{2} \pi / \partial m^{2}<0$ when $m<0$ so that there will be a unique optimal level of $m$ that is strictly less than zero.

(3) if $q^{\prime \prime}<0$ then $\partial^{2} \pi / \partial m^{2}<0$ when $m>0$, and $\partial^{2} \pi / \partial m^{2}>0$ when $m<0$. This means that it is always desirable to set $m$ as low as possible. For example, if 'bill and keep' or $m=-c_{0} / c$ is the lowest feasible value of $m$ then this will be the optimal value of the termination mark-up.

On the profit-side, note that as $m$ falls below zero, profits increase. Hence, $\pi_{i}>\pi^{H}$.

The intuition for this result is simple. When $m$ is negative, customers wish to belong to the smaller network, all else being equal. This is because inter-network calls will be priced below intra-network calls. As a result, lowering $F_{i}$ to attract marginal customers becomes a less viable competitive tool for networks, and price competition is muted. This softening of price competition increases equilibrium profits for both networks. ${ }^{5}$

A 'bill and keep' or 'peering' arrangement involves setting $m=-c_{0} / c$. In this situation, interconnect charges are zero with no settlement taking place; calls are billed but termination charges are kept. Proposition 2 demonstrates that the negotiated interconnect charge will be below marginal termination cost so that each network makes losses on interconnect. One simple way of achieving this is to set $m=-c_{0} / c$. This would save on accounting and other transaction costs. However, the profit maximising outcome may be greater or less than this. Nonetheless, the proposition does rationalise a 'bill and keep' arrangement as being consistent with collusive outcomes rather than in spite of them.

\footnotetext{
5 Proposition 5 of Laffont, Rey and Tirole (1998b) comes to a different conclusion than we do in this paper. That proposition states that the joint profit maximising level of $m$ is 0 and maximum profits will be achieved at the Hotelling level. While they prove that equilibrium profits are decreasing in $m$, they erroneously draw the conclusion that the lowest feasible level of $m$ is zero, claiming that the first order condition for profit maximisation is: $v((1+m) c)=v(c)+m c v^{\prime}((1+m) c)$ rather than the equation listed in the proof of Proposition 2 .
} 
An immediate implication of our analysis is that bill and keep termination charging, with the resulting low price of inter-network calls, may be undesirable from a consumers' perspective. While some call prices are low compared with, say, the non-cooperative equilibrium, this is offset by increased fixed charges. In particular, total value created is lower under bill and keep than if termination charges were set at cost, and network profits are higher. Thus, consumers are necessarily worse off under bill and keep or under any other co-operative equilibrium where termination charges are set below cost, than when termination prices are set at marginal cost.

\section{Conclusion}

Current research into interconnect arrangements between networks has argued that high charges will be used to soften network competition. Here we demonstrate that in an important class of network competition - namely, with two-part tariffs and price discrimination - that low, rather than high, interconnect charges can be used to soften price competition among networks. This is because low charges reduce the overall benefits of attracting a customer and hence, the intensity of bidding for subscribers. Consequently, it is possible that the often-used 'bill and keep' arrangements may perform a collusive function as well as minimising accounting and transaction costs.

The model here follows the literature in being essentially static. As such, there is no repeated element to networks' interactions. If this were to be examined, it is possible that low interconnect charges could sustain collusive outcomes in other market environments; including those with linear call pricing. In this situation, the low interconnect charges would make price wars more damaging to competitors and hence, commitments to them could help sustain tacitly collusive outcomes. However, 
a closer examination of this distinct role for low interconnect charges awaits further research.

\section{References}

Armstrong, M. (1998), "Network Interconnection in Telecommunications," Economic Journal, 108 (May), pp.545-564.

Carter, M. and J. Wright (1999), "Interconnection in Network Industries," Review of Industrial Organization, 14 (1), pp.1-25.

Laffont, J-J., P. Rey and J. Tirole (1998a), "Network Competition I: Overview and Nondiscriminatory Pricing," RAND Journal of Economics, 29 (1), pp.1-37.

Laffont, J-J., P. Rey and J. Tirole (1998b), "Network Competition II: Price Discrimination," RAND Journal of Economics, 29 (1), pp.38-56.

Williams, P. (1995), "Local Network Competition: The Implications of Symmetry," Annual Industry Economics Conference Volume, Bureau of Industry Economics: Melbourne. 